\title{
Identification of Mass-Unbalance in Rotor of an Induction Motor Through Envelope Analysis of Motor Starting Current at no Load
}

\author{
S. K. Ahamed ${ }^{1}$, M. Mitra ${ }^{2}$, S. Sengupta ${ }^{2}$ and A. Sarkar ${ }^{3}$ \\ ${ }^{1}$ Govt. College of Engineering \& Textile Technology, Serampore, Hooghly, W.B., India \\ ${ }^{2}$ Dept. of Applied Physics, University of Calcutta, 92, APC Road, Kolkata - 700009, India \\ ${ }^{3} \mathrm{MCKV}$ institute of Engineering Howrah, PIN -711204, India
}

Received 20 November 2011; Accepted 15 July 2012

\begin{abstract}
This paper developed a new technique for identification of mass-unbalance in rotor of an induction motor through envelope analysis using motor starting current at no-load The unbalanced magnetic pull due to centrifugal force developed produces excessive vibration in the rotor as well in the stator. This magnetic pull is very high at the starting which moves the rotor in the whole air gap, resulting changes in the air gap flux distribution in the stator and in the rotor. This induces voltage and generation of new signature pattern of motor current. The present method overcomes the difficulty of FFT analysis at steady state due to spectral leakage as the starting current is very high even at no load. Though DWT analysis is producing good results for transient motor starting current analysis, but selection of mother wavelet is not an easy task, if not proper, may introduce serious error. In the present improved method, the wavelet selection is not an important criteria. Envelope is the argument of the complex analytic signal which is obtained by using original motor current as the real and its Hilbert transform as the imaginary part. Since Envelope analysis works on narrow band instantaneous low frequencies, for which DWT was performed to extract low frequencies below $50 \mathrm{~Hz}$. using higher order wavelet at higher level. Simultaneously this method has higher detectability and higher resolution and it can also deal with small data efficiently, so it can be used online as well as offline.. This method has been tested in a laboratory prototype.
\end{abstract}

Keywords: Envelope, Induction Motor, mass-unbalance, Wavelet\& DWT, Hilbert Transform, FFT, Instantaneous frequency, detailed co-efficients. Power Detail Energy(PDE)

\section{Introduction}

Uninterrupted operation on the production line is major demand in the present day automation system. Without preventive maintenance, it is impossible to achieve. Induction Motor being the major prime mover in the industries, it is essential to detect any irregularities in the motor components at early stages before going severe and forcing unscheduled costly break down which requires online continuous monitoring of motor health for preventive maintenance. This leads to the development of online condition based monitoring system (CBM).

Generally, two types faults are observed in induction motor.

1. Electrical faults: stator winding short and open circuit, broken rotor bar and end ring fault.

2. Mechanical Faults: bearing damage, rotor eccentricity, shaft misalignment and bending.

Besides, load line or supply side or other external faults may also occur.

Mass unbalance in rotor
Mass unbalance in rotor occurs due to unequal distribution of mass around the centre of rotation of the rotor for which centre of gravity of rotor does not coincide with centre of rotation. Unbalance may occur due to

- manufacturing defect

- Non symmetrical addition or subtraction of mass due to wear, corrosion or erosion etc.

- Internal misalignment, shaft bending due to thermal expansion.

Mass unbalance may be classified as

1. static unbalance - rotational axis and weight distribution axis of rotor are parallel but offset as shown in Figure 1.

2. couple unbalance - rotational axis and weight distribution axis of rotor intersect at the centre of the rotor as shown in Figure 2.

3. dynamic unbalance - rotational axis and weight distribution axis of rotor don't coincide,

a combination of coupling unbalance and static unbalance as shown in Figure 3. 


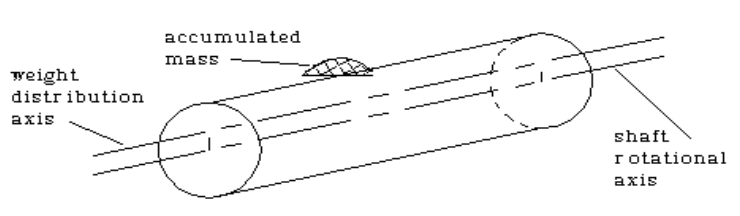

Fig. 1. Static unbalance

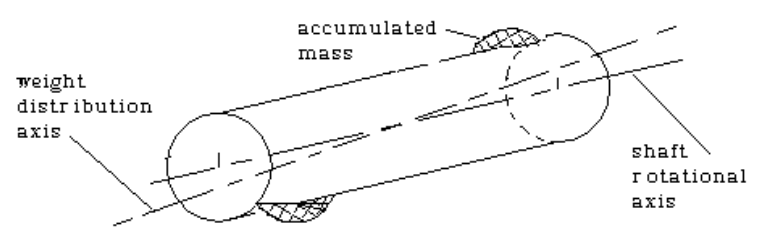

Fig. 2. Couple unbalance

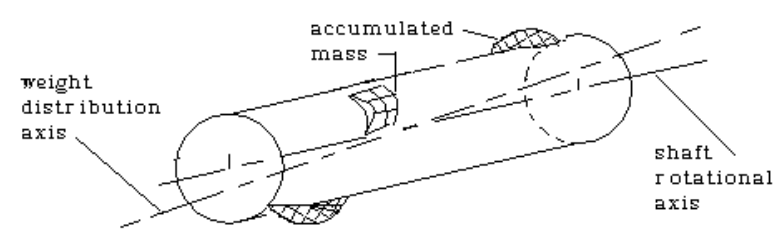

Fig. 3. Dynamic unbalance

Mass unbalance in rotor develops centrifugal force along with dynamic eccentricity. Total unbalance force is the sum of the centrifugal force and unbalance electromagnetic force. Rotor eccentricity increases due to this unbalance force which tries to pull apart the rotor further away from the stator bore centre, varying the air gap. This unbalance force changes with rotational speed [1]. During starting, the unbalance force is very high where the rotor oscillates through the whole air gap, may lead to bent rotor shaft, bearing wear and tear. If not detected, it may cause to rotor rub with the stator resulting catastrophic permanent damage of the motor.

The most widely used method for detection of motor asymmetry in rotor/stator is identification of side band harmonics or related fault harmonics by analysis of motor current signature (MCSA)at steady state through FFT technique [2 - 6] which is non-invasive. Other techniques used for fault detection are axial flux monitoring [7], acoustics [8] and vibration measurement[9],measurement of torque profile[10], voltage monitoring[4].This require special transducer or sensor to be fitted in and around the motor which is costly and delicate which interrupt the operation[11], besides these methods are also invasive. Due to power frequency leakage, side band harmonics or harmonics close to fundamental supply frequency are completely masked because of low amplitude, specially for small /medium power motor operating at light load or no load. To overcome these, scientists $\&$ researchers search for other signal processing techniques. Recently, wavelet transform are producing very good results specially for transient signals using starting motor current[12], starting vibration transient[9], motor current during speed and load changing[13] in which both time \& frequency informations remain intact instead of only frequency information in case of FFT analysis during steady state. But selection of mother wavelet is not an easy task as it is not a known priori. This may introduce serious error in the analysis. To overcome these constraints, in this paper a new technique has been presented through Envelope analysis using Hilbert transform and DWT.The concept of instantaneous frequency is introduced to find out the envelope.

The paper is organized as section 2 discusses the theoretical study - Theory of mass unbalance, Instantaneous frequency and Hilbert Transform, Envelope analysis and DWT. Proposed Technique was also been presented in this section. Section 3 discusses the experimentation details. Results and data analysis were presented in section 4 and the conclusion for this paper were made in section 5 .

\section{Theoretical Study}

\subsection{Theory}

During run of the motor, centrifugal force develops due to mass unbalance in rotor which increases with speed. This centrifugal force together with unbalanced electromagnetic force increases the dynamic eccentricity of the rotor which tries to pull apart the rotor further away from the stator bore centre, varying the air gap. During starting, the unbalance force is very high produces excessive vibration and in extreme case, it may lead to rotor rub with the stator resulting catastrophic permanent damage of the motor.

For healthy motor, the distribution of mutual inductances between stator and the rotor loops are symmetrical, but due to mass unbalance in rotor condition mutual inductances between stator and rotor loops become unsymmerical which induces characteristic harmonics as given by equation [1]

fault due to mass-unbalance in rotor creating dynamic

eccentricity, the stator current frequency is given by

$$
f_{u b m}=f\left[\frac{k(1-s)}{P}+1\right]
$$

where $\mathrm{P}$ is the no, of pole pairs, $\mathrm{s}$ is the slip and $\mathrm{f}$ is the supply frequency, $\mathrm{k}$ is an integer $=1,2,3,4 \ldots \ldots$

\subsection{Instantaneous frequency and Hilbert transform}

The periodic or sinusoidal frequency is not so much effective in analysis of transient / non-stationary signal for which the concept of instantaneous frequency has been introduced which gives better description of a transient. It is a time varying parameter which defines the spectral peaks varying with time. It is only applicable for mono component or narrow band frequency signals, defined as $f_{i}=(1 / 2 \Pi)$ $\mathrm{d} \Phi / \mathrm{dt}$ i.e. phase variability. Though the physical interpretation of instantaneous frequency is questionable [14], it can be only treated like periodic sinusoidal frequency if the corresponding signal can be represented in complex form like FM / AM signal as me ${ }^{j \Phi t}$ which can be done by Hilbert transform of $s(t)$, the original signal as Gabor and Ville noted which is described in the article[14]. The complex form of the signal is known as analytic signal. The 
unique complex representation of a real signal, $\mathrm{s}(\mathrm{t})$ is given by

$$
z(t)=s(t)+j[H[s(t)]
$$

The Hilbert transform is defined as

$$
H[s(t)]=\frac{1}{\pi} \int_{-\infty}^{\infty} \frac{x(\tau)}{(t-\tau)} d \tau
$$

Using the mean value theorem, we can evaluate

$$
H[s(t)]=\frac{1}{\pi t} \otimes s(t)
$$

Therefore, Hilbert transform is obtained if the original signal, $\mathrm{s}(\mathrm{t})$, is convolved with $1 /(\Pi t)$. Hilbert transform is equivalent to the positive frequencies from the spectrum of $\mathrm{s}(\mathrm{t})$ is shifted by $-\Pi / 2$ and their amplitudes are doubled and the negative frequencies are removed. The complex signal, $\mathrm{z}(\mathrm{t})$ is known as the analytic signal which does not always corresponds to the signal and its quadrature. If there is any significant leakage from positive frequencies to negative frequencies, Hilbert transform will not produce perfect quadrature component, analysis using this leads to ambiguous result.

If the signal is of the form, $\alpha(t) \cdot \cos \Phi(t)$ which is like a real FM signal and may be written as

$$
\begin{aligned}
& z(t)=s(t)+j H[s(t)]=a(\mathrm{t}) \cos \Phi(t)+ \\
& +j H[\alpha(t) \cos \Phi(t)] \\
& \text { Or, } z(t)=\alpha(t)[\cos \Phi(\mathrm{t})+j \sin \Phi(\mathrm{t})] \\
& \text { or, } z(t)=\alpha(t) e^{j \Phi(\mathrm{t})}
\end{aligned}
$$

The equation (5) indicates that the analytic signal is of the AM/ FM modulated signal. For significant meaningful practical feasibility, the amplitude spectra of $\alpha(\mathrm{t})$ which belongs to low frequency region and the phase spectra of $\Phi(\mathrm{t})$ which belongs to high frequency region to be separately considered. Our present interest is limited to low frequency amplitude spectra below supply frequency.

\subsection{Envelope analysis}

A new dimension for determining fault frequency.

The envelope of a complex signal, $z(t)$ is defined as

$$
E(t)=|s(t)+j H[s(t)]|
$$

or, $E(t)=\alpha(t)$

That is, the absolute value of the analytic signal defined in equation (5). The envelope signal occupies the low frequency spectral region, the analysis of which gives better detection than the spectrum analysis of the original signal as the power frequency is eliminated from the signal.

\subsection{Discrete Wavelet transform(DWT)}

DWT decomposes a sampled signal $\mathrm{s}(\mathrm{t})$ by passing it through HPF(high pass filter) and LPF(low pass filter) into its approximate signal $a_{n}$ and several detail signals $d_{j}[15]$ as given in equation[7]

$$
s(t)=\sum_{i} \alpha_{i}^{n} \varphi_{i}^{n}(t)+\sum_{j=1}^{n} \sum_{i} \beta_{i}^{j} \psi_{i}^{j}(t)=a_{n}+d_{n}+--+d_{1}
$$

Where $\alpha_{i}^{n}, \beta_{i}^{j}$ are scaling and wavelet function, $\varphi^{n}(t)$ is the scaling function at level $\mathrm{n}$ and $\psi^{j}(t)$ is the wavelet function at level $\mathrm{j} ; a_{n} \& d_{j}$ is approximate signal at decomposition level $\mathrm{n}$ and detail signal at level $\mathrm{j}$ (16).

Each step of decomposition of the signal corresponds to certain frequency resolution. Figure4 shows typical two level wave decomposition. At each level of scaling for various positions, the co-relation between signal and wavelet are called wavelet coefficients. High pass filter coefficients are called detail coefficient $\left(d_{n}\right)$ and low pass filter coefficients are called approximate coefficients $\left(a_{n}\right)$. The first level of decomposition coefficients are $a_{1}$ and $d_{1}$, where $\mathrm{a}_{1}$ is the approximate version of and $\mathrm{d}_{1}$ is the detailed version of the original signal. At each decomposition level, the corresponding detailed and approximate coefficients have definite frequency bandwidths given by $\left[0-\mathrm{fs} / 2^{1+1}\right]$ for approximate coefficient, $a_{1}$ and $\left[\mathrm{fs} / 2^{1+1}-\mathrm{fs} / 2^{1}\right]$ for detail one $d_{1}$ where $f_{s}$ is the sampling frequency, 1 denotes the decomposition level limited by the sampling frequency $f_{s}$, where $f_{s} / 2$ is the corresponding Nyquist frequency. At each step of decomposition the sampled dataset are downsampling by a factor of $2 \downarrow$, which is called dyadic decomposition.

After decomposition of the signal, one can examine the constituent components at each detail level. The spectral frequency bands of different detailed coefficients are shown in the Table 1.

Power Detail Energy:

It is defined as given in equation (8)

$P D E=\sum_{1}^{N}\left(d_{j} \times d_{j}\right)$

Where $P D E$ is the detail energy concentrated at level $j$, containing no. samples N.

\subsection{Proposed technique}

The present technique develops a new innovative method for detection of mass unbalance in rotor through envelope analysis of the motor current signal at no load using higher order discrete wavelet at higher level by extracting low frequency oscillation below supply frequency 50 cycles/ sec. Envelope analysis is based on the principle of instantaneous 
frequency which is applicable for mono component or signals of narrow band frequencies and wavelet at higher level belongs to the narrow band zone, free from overlapping bands and thus minimizes the possible leakage is excellent choice for the present analysis. Envelope is the low frequency amplitude spectra in which supply frequency is completely eliminated which makes the analysis most effective. Higher order wavelet for smoothness [17] i.e. to avoid non-ideal characteristics which appears at lower order though selection of mother wavelet is not at all a problem in envelope analysis is very much selective in efficiently filtering the narrow band frequencies below power supply frequency in its higher wavelet level as given in Table-1. At higher level, the amplitude of original current signal is very low, but for envelope, the amplitude of the spectral bands are very high which works on the principle of instantaneous frequency. Envelope is the absolute value of the analytic signal obtained as $E(t))=|z(t)|=|s(t)+j H[s(t)]|$ where $s(t)$ is the real signal and $\mathrm{H}[\mathrm{s}(\mathrm{t})]$ is its Hilbert transform which is in quadrature with the real signal, $z(t)$ is the analytic signal. As motor current signal is sinusoidal, will be expressed in the complex form as $z(t)=\alpha(t) e^{j \Phi(t)}$ as given in (5) which is like FM /AM signal. The amplitude part $\alpha(t)$ which is the envelope belongs to the low frequency instantaneous zone can be analyzed like periodic frequency. Detail coefficients, Power Detail Energy (PDE) are considered as the fault parameters which is compared with healthy motor for identification. This technique provides an excellent method for identifying mass unbalance in rotor using transient startup current at no load by extracting low frequency oscillation through envelope analysis.

Table 1. Spectral Frequency Bands at different decomposition levels

\begin{tabular}{l|c}
\hline Decomposition Details & Frequency Bands(Hz) \\
\hline Detail Level 1 & $2048-4096$ \\
\hline Detail Level 2 & $1024-2048$ \\
\hline Detail Level 3 & $512-1024$ \\
\hline Detail Level 4 & $256-512$ \\
\hline Detail Level 5 & $128-256$ \\
\hline Detail Level 6 & $64-128$ \\
\hline Detail Level 7 & $32-64$ \\
\hline Detail Level 8 & $16-32$ \\
\hline Detail Level 9 & $8-16$ \\
\hline Detail Level 10 & $4-8$ \\
\hline
\end{tabular}

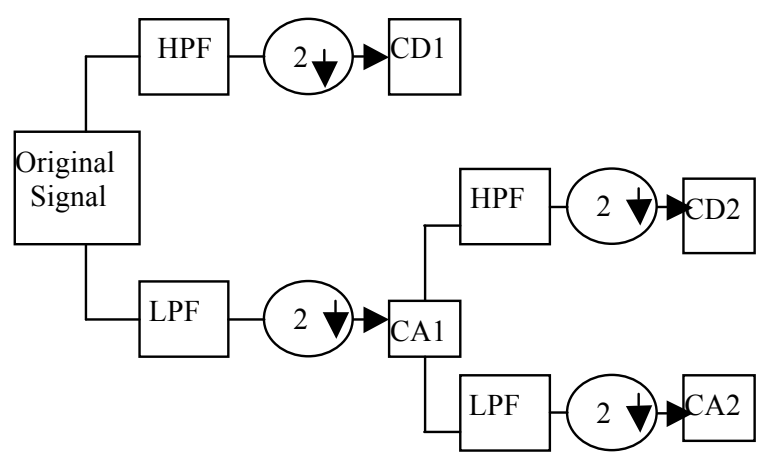

\section{Experimentation}

\subsection{Block Diagram}

The block diagram is shown in Figure 5. $3 \mathrm{ph}, 110 \mathrm{~V}, 50 \mathrm{~Hz}$, supply is provided to the two induction motors of same rating one healthy, the other one with unknown mass unbalance in rotor, last one being intentionally made, faulty motor. The motors are run by direct-on-line supply.

\subsection{Experimental Setup}

Experiment was carried out on test-rig built by Spectra Quest, USA, having a high speed data acquisition system (OROS OR35, 8 channels, $100 \mathrm{mbps}$ ). Rating of the induction motors are $3 \mathrm{ph}, 1 / 3 \mathrm{HP}, 190 \mathrm{~V}, 50 \mathrm{~Hz}, 2980 \mathrm{rpm}$. For capturing current signature Hall Probe (LEM PR30 ACV $600 \mathrm{~V}$ CATIII 30Ampac/3Vac) is used. The experimental set up is shown in Figure 6.Transient current envelope has been captured with a sampling frequency of $8.192 \mathrm{kc} / \mathrm{sec}$.

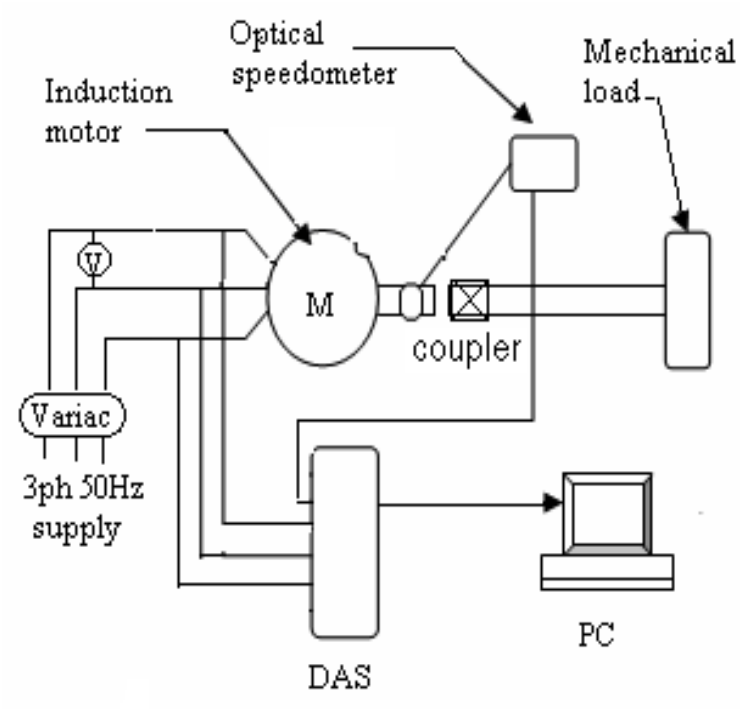

Fig. 5 Block diagram of the experiment

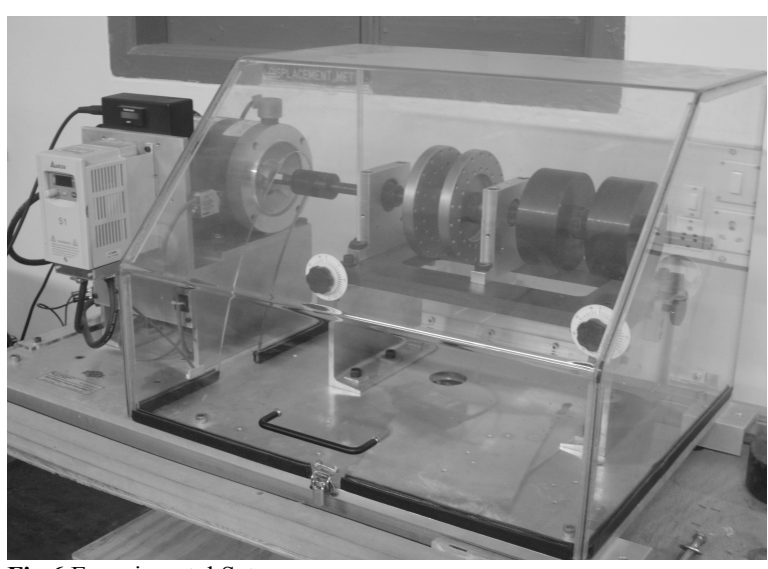

Fig.6 Experimental Setup

Fig.4 Two Level DWT decomposition 


\section{Results and data analysis}

The proposed technique has been applied in the laboratory prototype for detection of induction motor mass unbalance in rotor fault. Two identical 3 ph induction motors, one healthy and the other with mass unbalance in rotor of rating 1/3 H.P., $190 \mathrm{~V}, 50 \mathrm{hz}, 2980 \mathrm{rpm}$ were used. The starting current signatures for both the motors were captured using Hall probe sensors (LEM PR30 ACV 600V CATIII 30ampac / 3 Vac) at a sampling frequency of 8.192 $\mathrm{kc} / \mathrm{s}$. The block diagram and experimental setup are given in Figure 5 and Figure 6 . Then the signal envelopes were extracted from the captured motor current signatures for both the motors at no load (shown in Figures 7, 8 ) and decomposed into details and approximate coefficients through DWT using "db10"of Daubechies family upto wavelet level 10. The signal envelopes are the absolute value i.e. the argument of the analytical signal obtained from the HT of the original signal. The decomposed details at $8^{\text {th }}, 9^{\text {th }}$, $10^{\text {th }}$ levels for both the motors are given in figures 9,10 .

The data analysis is performed on Matlab platform. In this method higher order wavelet " $\mathrm{db} 10$ " is used to extract lower side harmonics below $50 \mathrm{~Hz}$ at higher levels $8^{\text {th }}, 9^{\text {th }}$ and $10^{\text {th }}$. Then the detail coefficients are processed to determine power detail energy known as PDE of the envelope and statistical parameters - the mean, the standard deviation and r.m.s. of the detail coefficients of the starting current signal envelopes for both the motors at no load are estimated. Here statistical parameters and PDE are considered as fault parameters. In the envelope signal, power supply frequency is completely eliminated which makes the detection more cleaner and effective. From the table 2 and curves as given in Figures 11, 12,13 it is observed that $8^{\text {th }}$, $9^{\text {th }}, 10$ th levels indicate higher mean values of detail coefficients of the faulty motor with respect to the healthy motor, specially at $9^{\text {th }}$ and $10^{\text {th }}$ level, it is considerably higher and Mass unbalance in rotor motor yields higher standard deviation and r.m.s values of detailed co-efficients at $8^{\text {th }}, 10^{\text {th }}$,specially at 10 th level, the values are very much higher as shown encircled whereas at $9^{\text {th }}$ level value is lower than the healthy motor which does not conform to the expected results. In the energy side, power detail energy (PDE) of the envelopes give same trend of results as obtained in the case of standard deviation and r.m.s. values of the detail coefficients i.e. $8^{\text {th }}, 10^{\text {th }}$ level indicates higher energy consumption, specially very higher enhancement of energy at the 10th level as shown encirled whereas at the $9^{\text {th }}$ level, it is lower for mass unbalance in rotor motor than the healthy motor as revealed from the curve in Figure 14 and Table3. The signal envelope at no load produces higher values of the statistical standard deviation of and r.m.s. values of the decomposed details for faulty motor at $8^{\text {th }}$ and $10^{\text {th }}$ wavelet levels than those of the healthy motor whereas statistical means are higher at all these three levels for the faulty motor. In the analysis of energy parameter, the values of PDE is higher at $8^{\text {th }}$ and $10^{\text {th }}$ wavelet levels ,particularly at 10th level, very sharp increase of the value of PDE for the faulty motor with respect to the healthy motor is observed. Therefore, it can be inferred from the above experimental data analysis that the $10^{\text {th }}$ level is most sensitive to detect fault (mass unbalance in rotor) at no load.

From the above observations, it is clear that the present method using envelope analysis is quite efficient to extract low frequency oscillations below $50 \mathrm{hz}$ from starting current at no load for detection of mass unbalance in rotor fault. As the power frequency is completely eliminated from the envelope, spectral leakage is avoided which improves the detectability and due to application of higher order wavelet at higher level, the method works with higher resolution

The main drawbacks using this method is once the sampling frequency selected, the spectral bands become fixed which means some ranges of frequencies are unexplored. In the present analysis, the range - ( $32 \mathrm{hz}-50$ $\mathrm{hz}$ ).

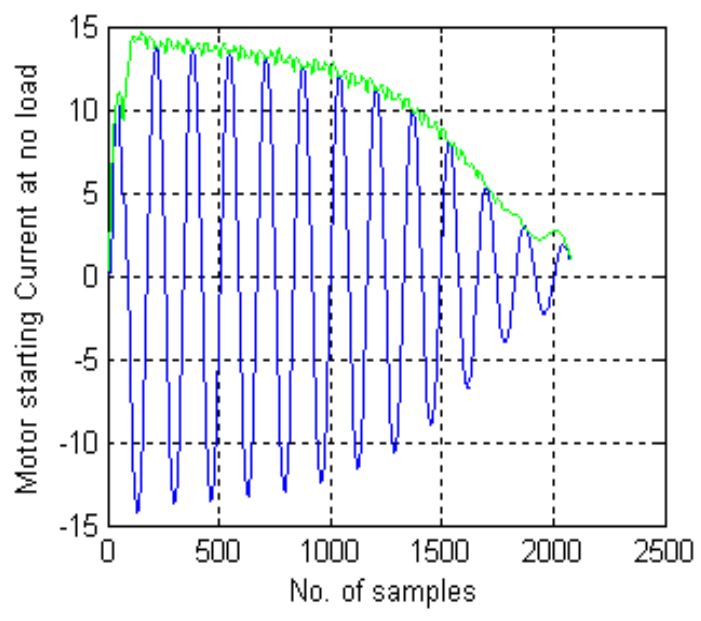

Fig. 7 Healthy signal and its envelope at no load

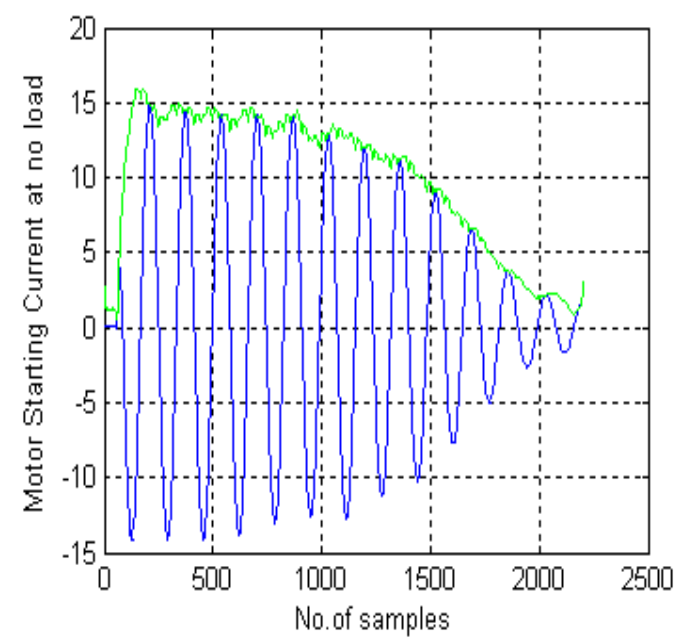

Fig. 8 Faulty signal and its envelope at no load
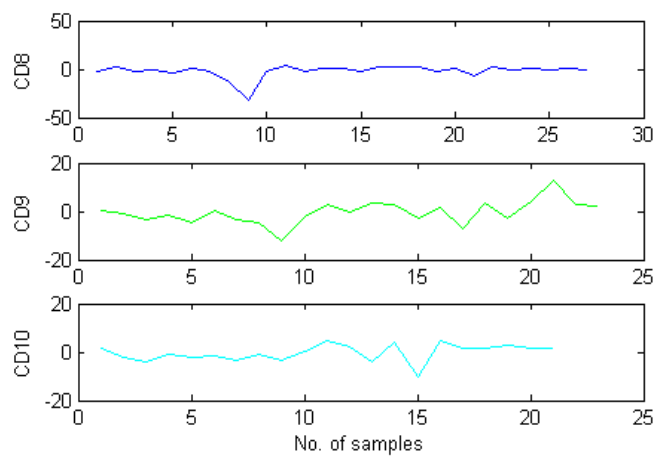

Fig. 9 Detail Co-efficients of Motor (Healthy) Starting Current signal Envelope at no-load 

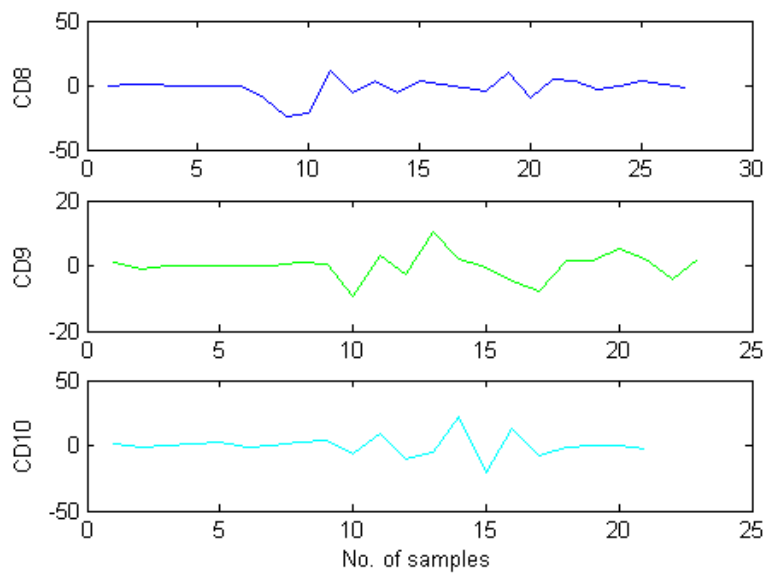

Fig. 10 Detail Co-efficients of Motor (Faulty) Starting Current signal Envelope at no-load

Table 2. Statistical parameters of Detail Co-efficients of Motor Starting Current Signal Envelope at no-load

\begin{tabular}{llcc}
\hline \multicolumn{1}{c}{ Motor Condition } & \multicolumn{2}{c}{ PDE at Wavelet Level in Joules } \\
\cline { 2 - 4 } & 8 & 9 & 10 \\
\hline Healthy & 1356.000 & 509.843 & 260.044 \\
\hline Faulty & 1704.000 & 357.478 & 1464.9 \\
\hline
\end{tabular}

Table 3. Power Detail Energy of Motor Starting Current Signal Envelope at no-load

\begin{tabular}{|c|c|c|c|c|c|c|c|c|c|}
\hline \multirow[t]{2}{*}{$\begin{array}{l}\text { Motor } \\
\text { Condit } \\
\text { ion }\end{array}$} & \multicolumn{3}{|c|}{$\begin{array}{l}\text { Mean of Detail } \\
\text { Coefficient } \\
\text { at Wavelet Level }\end{array}$} & \multicolumn{3}{|c|}{$\begin{array}{c}\text { Standard } \\
\text { Deviation of } \\
\text { Detail Coefficient } \\
\text { at Wavelet Level }\end{array}$} & \multicolumn{3}{|c|}{$\begin{array}{c}\text { RMS of Detail } \\
\text { Coefficient } \\
\text { at Wavelet Level }\end{array}$} \\
\hline & 8 & 9 & 10 & 8 & 9 & 10 & 8 & 9 & 10 \\
\hline $\begin{array}{c}\text { Health } \\
\mathrm{y}\end{array}$ & $\begin{array}{c}- \\
1.9 \\
44\end{array}$ & $\begin{array}{c}- \\
0.40 \\
21\end{array}$ & $\begin{array}{c}- \\
0.25 \\
66\end{array}$ & $\begin{array}{l}6.9 \\
45\end{array}$ & $\begin{array}{l}4.7 \\
96\end{array}$ & $\begin{array}{l}3.5 \\
96\end{array}$ & $\begin{array}{c}7.08 \\
60\end{array}$ & $\begin{array}{c}4.70 \\
82\end{array}$ & $\begin{array}{c}3.51 \\
90\end{array}$ \\
\hline Faulty & $\begin{array}{c}- \\
1.8 \\
97\end{array}$ & $\begin{array}{c}0.00 \\
97\end{array}$ & $\begin{array}{c}0.01 \\
72\end{array}$ & $\begin{array}{l}7.8 \\
62\end{array}$ & $\begin{array}{l}4.0 \\
31\end{array}$ & $\begin{array}{c}8.5 \\
58\end{array}$ & $\begin{array}{c}7.94 \\
44\end{array}$ & $\begin{array}{c}3.94 \\
24\end{array}$ & $\begin{array}{c}8.35 \\
20\end{array}$ \\
\hline
\end{tabular}

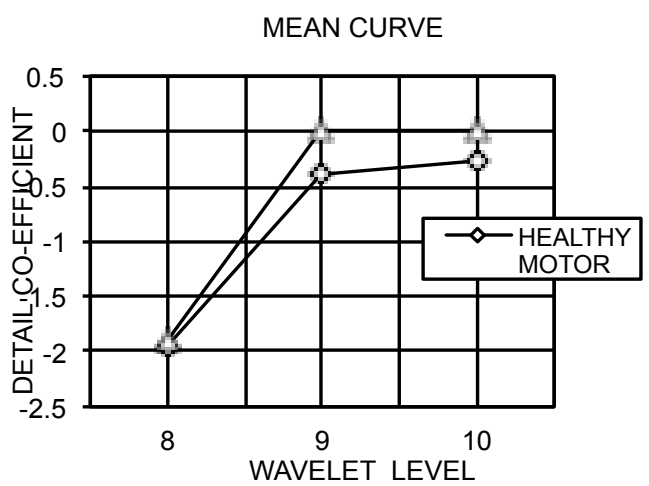

Fig. 11. Mean Curve

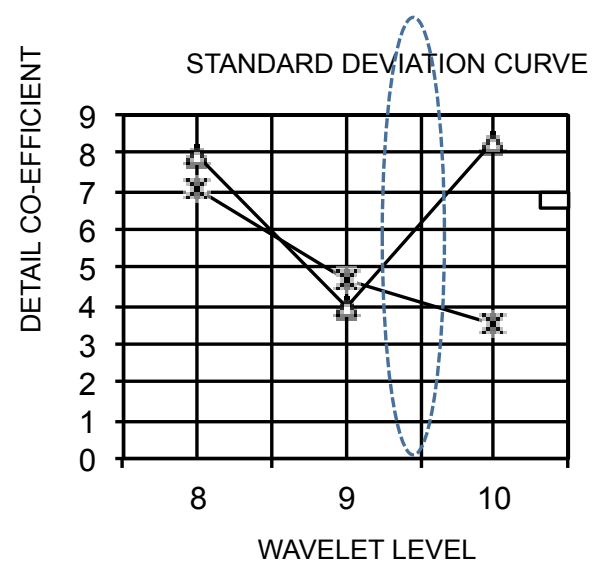

Fig. 12. Standard Deviation Curve

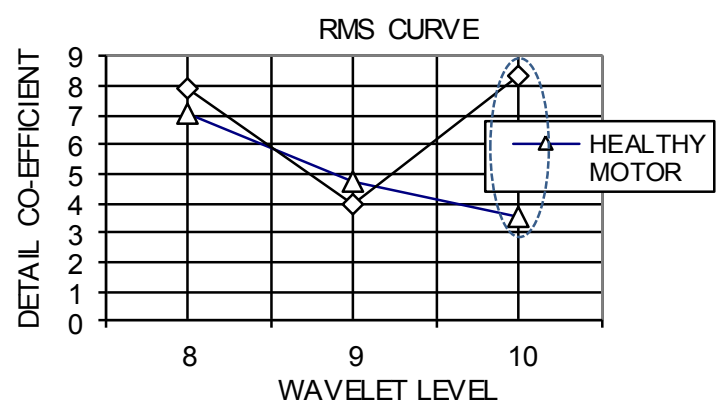

Fig. 13. RMS Curve

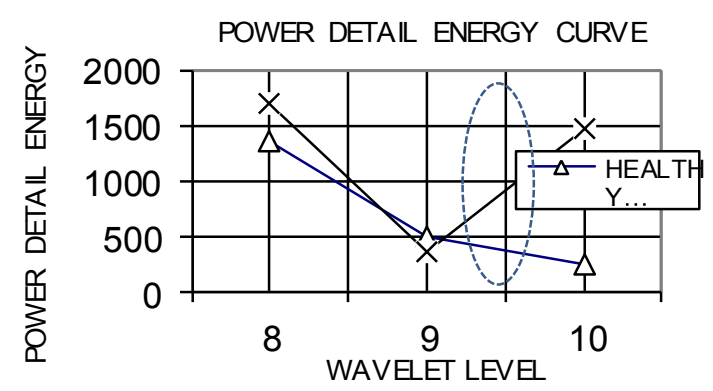

Fig. 14. Power Detail Energy Curve

\section{Conclusion}

The present technique offers a new diagnostic methodology which extracts low frequency oscillation below $50 \mathrm{hz}$ from the starting current at no load using envelope analysis which is free from spectral leakage due to elimination of $50 \mathrm{hz}$ from the signal. It works with higher detectability and higher resolution. As the no of computation is also less and no complex software required compared to FFT and other time frequency tools, the method is very suitable for online analysis in the present day industry. The technique may be applied to other faults analysis. 


\section{References}

1. Han,D.C. and H.J.M. "Lateral vibration analysis of the rotor system with magnetic forces," Proc. Of the $16^{\text {th }}$ Intern. Symp. On Transport Phenomena and Dynamics of Rotating Machinery, vol.1, pp. 87- 96, 1996.

2. Bellini's A., Filippetti, F., Franceschini, G., Tassoni, C. "Closed-loop control impact on the diagnosis of induction motors faults, Industry Applications ", IEEE Transactions 36 (5), pp .1318 - 1329, 2000.

3. Bellini A., Filippetti F., Franceschini G., Tassoni C. and Kliman G.B. "Quantitative evaluation of induction motor broken bars by means of electrical signature analysis", IEEE Transactions on Industrial Appl., 2001, 37, pp. 1248-55.

4. Tallam, R . M, Habetler, T.G.,Harley, R.G.,"Stator winding Turn- fault detection for closed-loop induction motor drive, Industry Applications ", IEEE Transactions 39 (3), pp . $720-724,2003$.

5. Didier, G., Ternicien ,E., Caspery, O., and Rajik, H., "Fault detection of broken rotor bars in induction motor using a Global fault index ", Industry Applications IEEE Trans. Ins. Appl . vol . 42, no. 1, pp . 79 - 88, Jan. / Feb. 2006.

6. Kia , S . H., Henao, H., Capolino, G-A.), "A HighResolution Frequency Estimation method for ThreePhase Induction Machine Fault Detection, Industrial Electronics", IEEE Transactions 54 (4), 2305 - 2314 , 2007.

7. Assaf, T., Henao, H., Capolino, G.-A., "Simplified Axial Flux Spectrum Method to Detect Incipient Stator Inter-turn Short-Circuits in Induction Machine", IEEE, 0-7803-8304-4/4, 2004.

8. Wu, R-C., Tsai, J-I, Chiang, C-T. , Ouyang, C-S., "Detection of Induction Motor Operation Condition by Acoustic Signal", IEEE, 978-1-4244 -7300- 7/10, 2010.

9. Rodrigruez - Donate, C., Romero - Troncoso , R. J., Garcia-Perez A ., Razo - Montes, D. A.,"FPGA based embedded system for induction motor failure monitoring at the start up transient vibrations with wavelets ", IEEE, $978-1$ - 4244 - 1995 - 1/08, 2008.

10.Liang,B., Payne, B.S., Ball, A.D., Iwnicki, S. D.,"Simulation and fault Detection of three-phase induction motors", Mathematisc and Computers In simulation 61, 1-15, 2002

11. Watson, J.F., Paterson, N. C. , "Improved Techniques for Rotor Fault Detection in three phase Induction Motors", 0 - 7803 - 4943 - 1/98 . 1998, IEEE.

12. Supangat, R., Ertugrul,N., Soong, W.L., Gray, D.A., C. Hansen, Griegar, J. (2006), "Detection of Broken Rotor bars in Induction Motor using Starting Current Analysis and Effects of Loading ", IEE Proc.-Electr. Power Appl, Vol. 153, No.6, November 2006.

13. Cusido, J. , Romeral, L., Ortega, J. A. , Rosero, J. A.,"Fault Detection in Induction Machines Using Power Spectral Density in Wavelet Decomposition", IEEE Transaction on Industrial Electronics, vol . 55, no.2, February, 2008.

14. Boulam, B., "Estimating and Interpreting The Instantaneous Frequency of a Signal- Part1: Fundamentals", Proceedings of the IEEE, vol. 80, no. 4 , April 1992.

15. Antonino - Daviu , J. , Reira - Guasp , M., RogerFolch, J., Molina, M. P., "Validation of a new method for the Diagnosis of rotor bar failures via wavelet transformation in Industrial induction machines", IEEE Trans . Ind. Appl ., vol. 42 , no . 4 pp. 990 - 996, Jul. / Aug 2006.

16. Polikar,R., Udpa,L., Udpa, S.S., Taylor,T., "Frequency Invariant Classification of Weld Inspection Signals", IEEE Trans . Ultra Ferro and Freq. Cont., vol. 45 , no .3, pp. 614 - 625, 1998.

17. Douglas H., Pillay P., "The Impact of Wavelet Selection on Transient Motor Current Signature Analysis“, 0-7803-8987-5/05 2005,IEEE. 